Frank ZenKeR

\title{
SIMILARITY AS DISTANCE: THREE MODELS FOR SCIENTIFIC CONCEPTUAL KNOWLEDGE
}

\section{Introduction}

Starting with Minsky (1975) and more widely recognized since Barsalou (1992), (dynamic) frames have become a well-accepted tool to model conceptual knowledge. We begin our comparison with its predecessor model, the feature list, and trace its development into the (dynamic) frame model. Subsequently, we summarize extant frame-style reconstructions of taxonomic change as a paradigmatic application within history and philosophy of science, thus addressing the incommensurability of frameworks, or world-views, from a cognitive historical perspective.

We hold that a frame is a sophisticated feature list whose application centrally supports the claim that historically successive taxonomies are comparable. But we criticize that the frame model yields little insight beyond taxonomic change. Introducing conceptual spaces as an alternative model, we discuss dimensions, their combinations, how to recover frames by analogue expressions, similarity as geometric distance across diachronically varying spaces, and the status of scientific laws.

\section{Feature lists, frames, spaces}

Originating in Aristotelian philosophy (Taylor, 2003), the feature list constitutes both an entrenched and a somewhat outdated tool for reconstructing conceptual knowledge. Paradigmatically instantiated by taxonomic knowledge, 
e.g., in biology, it can be summarized by three assumptions: (i) the representation of a concept provides a summary of the class of its instances (i.e., the things falling under the concept); (ii) the binary features representing a concept are individually necessary and jointly sufficient to define the concept; (iii) features are nested in subset relations such that, if concept $X$ is a subset of concept $Y$, then the defining features of $\mathrm{Y}$ are nested in those of $\mathrm{X}$. Such features are regularly referred to as defining or essential (see Kuukkanen, 2006: 88).

On the classical view, combinations of binary features (aka attributes) provide a definition of that which falls under, or instantiates, a concept if and only if the features are present in, or true of, the thing. Features are normally rendered in natural language, typically by nouns or adjectives. The classical example sees MAN being analyzed as [+biped, + rational, +animal]. Standardly, problems arise upon observing, for instance, that a Para-Olympic athlete may thus fail to instantiate MAN - which is somewhat absurd. Short of allowing ad hoc modifications, or throwing individually necessary and jointly sufficient features over board, however, the absurdity is not easily remedied. The model's merits are worlds neatly cut along the patterns that such features generate, where the choice of features may always be a matter of convention, and particular conventions may be contingent upon contexts. To categorize champagne, vodka, fruit juice, and soda water, for instance, why not borrow from chemistry and use $\left[+/-\mathrm{C}_{6}\right]$ alcohol, $\left[+/-\mathrm{CO}_{2}\right]$ carbon-dioxide.

Barsalou and Hale (1993) demonstrate that feature lists contain rich relational information, primarily with respect to truth, as attributes count as true or false of a thing. Secondly, whatever a feature names, if true of the thing, will name one of its aspects. Thirdly, as set-members, a concept's defining features obey the logical relation of conjunction, just as several concepts obey exclusive disjunction. Fourthly, contingent relational information may be read off a feature list, thus allowing for strict or probabilistic predictions such as: consumers of items in the $+\mathrm{C}_{6}$ category (likely) need a designated driver. Finally, nesting of concepts accounts for the analytic character of "A bachelor is a man", as BACHELOR, when analyzed as [+man], [-married], is subordinate to MAN.

Exemplar and (weighed) prototype models are mathematically refined extensions of the feature list model that seek to remedy the absurdity of the Para-Olympic example, above. They are in part motivated also by empirical investigations into human categorization (Labov, 1973; Rosch et al., 1976; see Jaeger, 2010) which strongly suggest that humans do not, invariably across con- 
texts, categorize via necessary and sufficient features. Whether all models operate at the symbolic level, i.e., presuppose an explicit language, may be debated. Such refined models, at any rate, remain grounded in feature lists, but abandon the strictness with which the (possibly weighed) presence of features projects into category-membership. So in principle, but see below, considerations of similarity rather than identity may govern concept boundaries (see Barsalou, Hale, 1993: 103-124).

The above relations give rise to the frame model of concepts, introduced in the next subsection. Generally, the frame model qualifies as an extension of the traditional feature list, reached by allowing non-binary features (such as large, medium, small) and the relations of constraint and invariance.

\subsection{A frame is a sophisticated feature list}

When one suspends the additional elements introduced by frames, and moreover restricts attributes to binary values, then the frame model collapses into the feature list model, rather than approximating some model analogous to it. This should become clear when appreciating that frames may be stepwise generated from feature lists.

The first step beyond feature lists requires understanding a feature as the value of some attribute. For example, [+blue], [+green] are binary values of the attribute "color" and [+long], [+round] are binary values of the attribute "shape". The additional structure (above that of feature lists) consists in using more than two values to define an attribute, i.e., allowing such values to be $n$-ary. Therefore, an additional relation (which a feature list model does not allow to represent) is that between an attribute and its value(s), called the type-relation (informally: the is-a relation) such as "square" is a type of shape, "blue" is a color, etc. In a second step, one takes attributes to display structural invariants which "specify relations between attributes that do not vary often across instances of a concept" (Barsalou, Hale, 1993: 125). Moreover, and in contrast, constraints represent relations between attribute values "which instead vary widely across the instances of a concept" (ibid.: 125). One thus reaches the notion of a simple frame, defined as "a co-occurring set of multi-valued attributes that are integrated by structural invariants" (ibid.: 126), such that constraints hold across values and "produce systematic variability in attribute 
values" (Barsalou, 1992: 37). For instance, a comparatively slim person will normally not be heavy (relative to her height). In combination with structural invariants, then, constraints generate structure for the purpose of representing a concept(-instance), giving rise to the notion frame-pattern. Both constraints and structural invariants take on a pivotal role in reconstructing scientific conceptual change (see below).

The main advantage that the frame model can so far claim over feature lists is that "the addition of 'attribute-value relations' and 'structural invariants' increases their expressiveness substantially" (ibid.: 127), once given the means to model both stable and variable relations across attributes and values. Consequently, one may view the representation of a concept to proceed primarily via structural invariants and constraints. Structural invariants, as it were, tell us which attributes (are likely to) collect, or bind, into a concept, and constrained values identify concept instances.

In a final step, by recursion, the components used in conceptual representation (i.e., attributes, values, structural invariants and constraints) are taken to be represented not by linguistic means ("words"), but by frames: "[T]his recursive process can continue indefinitely, with the components of these more specific frames being represented in turn by frames themselves" (ibid.: 133). Whenever conceptual knowledge shall include not just things, but also relations (such as is-part-of, or requires), again, frames are employed recursively: "At any level of analysis, for any frame component, there is always the potential to note new variability across exemplars of the component and capture it in a still more specific frame" (ibid.: 134). So there is no principled limit to finding new attributes, "simply by noting variance across the component's exemplars and representing this variance with a new attribute-value structure" (ibid.: 133f.). Such recursion results in the more specific structure representing the less specific one, while retaining a symbolic representation nevertheless. After all, both attributes and values are primarily identified through natural language terms. We return to this aspect when comparing frames to conceptual spaces, which is a non-symbolic model, below.

A frame's attributes and values normally arise empirically from querying experimental participants. The choice of attributes, supposedly, is always influenced by "goals, experience and intuitive theories" (Barsalou, 1992: 34). The examples discussed in the literature therefore count as partial representations, including event frames (aka scripts), which are sequential adaptations of the ob- 
ject-frames discussed here. In the scientific case, the identification of attributes, values, etc. is based on the material under study, and so will draw on science historical work, but itself constitutes a more a systematic contribution to the philosophy of science.

\subsection{Applying frames to taxonomic change}

To further appreciate the frame model, a simple example from ornithology may be helpful. Based on Chen (2002), it does without iteration, employs binary features, and concerns the late 18th century discovery of a South American species of bird (commonly called "screamer") that "has webbed feet like ducks but a pointed beak like chickens" (ibid.: 7). This particular combination of features was "not allowed", and so constituted an anomaly to the then-standard taxonomy of Ray (1678). The misfit violated the constraint that the attributes foot and beak always go together. "This anomaly forced [ornithologists] to alter the frame of bird and the associated taxonomy, because it made a very important constraint relation between foot and beak invalid" (Chen, 2002: 7). Figures 1 and 2 illustrate partial frames of the predecessor taxonomy by Ray (1678), and the revised taxonomy by Sundevall (1889), respectively. Notice that, to distinguish WATER from LAND-BIRD, Ray's taxonomy employs the attributes beak (values: round or pointed) and foot (webbed or clawed), connected by a structural invariant (double-headed arrow); see Chen (2002: 5).

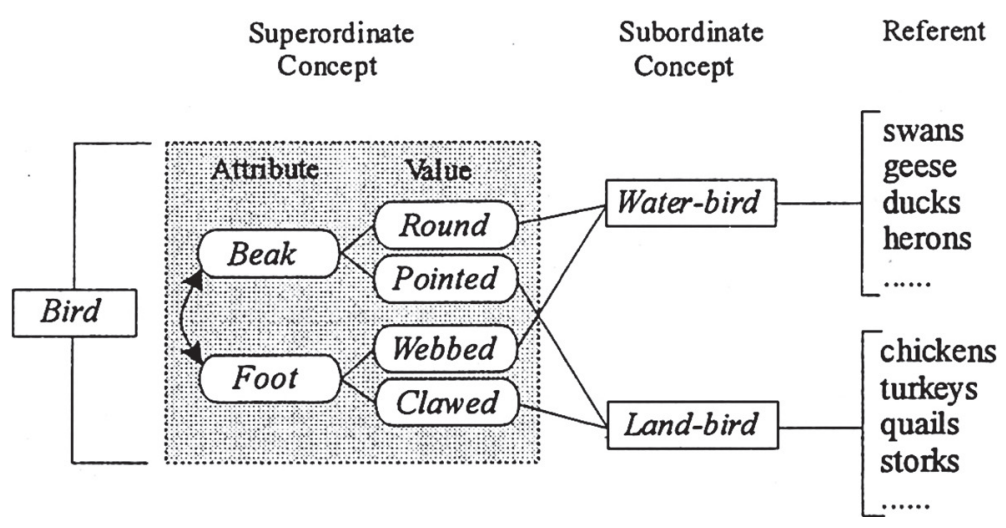

Fig. 1. Partial frame for Ray’s (1678) bird concept (Chen, 2002) 


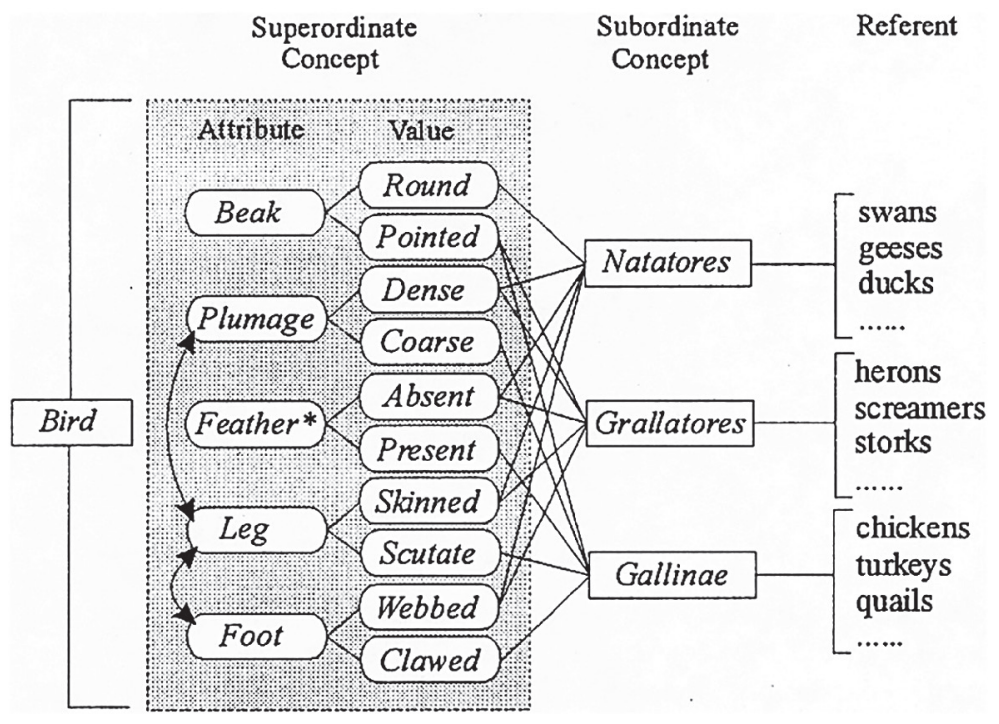

Fig. 2. Partial frame for Sundevall's (1889) bird concept (Chen, 2002)

The historical transition between the representations in figures 1 and 2 is standardly interpreted as a redefinition of the concept BIRD. To frame-theorists, moreover, the example demonstrates scientific taxonomic change to be reconstructable as a motivated revision.

Sundevall's bird no longer entails a constraint relation between beak and foot; instead, new constraint relations are formed between foot and plumage, as well as between foot and leg covering. $[\mathrm{T}]$ hese are physical constraints imposed by nature, resulting from the adaptation to the environment. The new superordinate concept inevitably alters the taxonomy by expanding the conceptual field at the subordinate level. (Chen, 2002: 8)

Further contrasting fig. 2 with the successor taxonomy developed by Gadow (1893), in fig. 3, the subsequent transition to it may be interpreted as a more radical shift than that from Ray's to Sundevall's. After all, "Darwin discovered that species are not constant, and therefore affinity among species must be founded on their common origin" (Chen, 2002: 12). Gadow's taxonomy, moreover, had been developed in response to Sundevall's which "emphasized the dissimilarities between screamers and waterfowl” (ibid.: 12) rather than their similarities. 


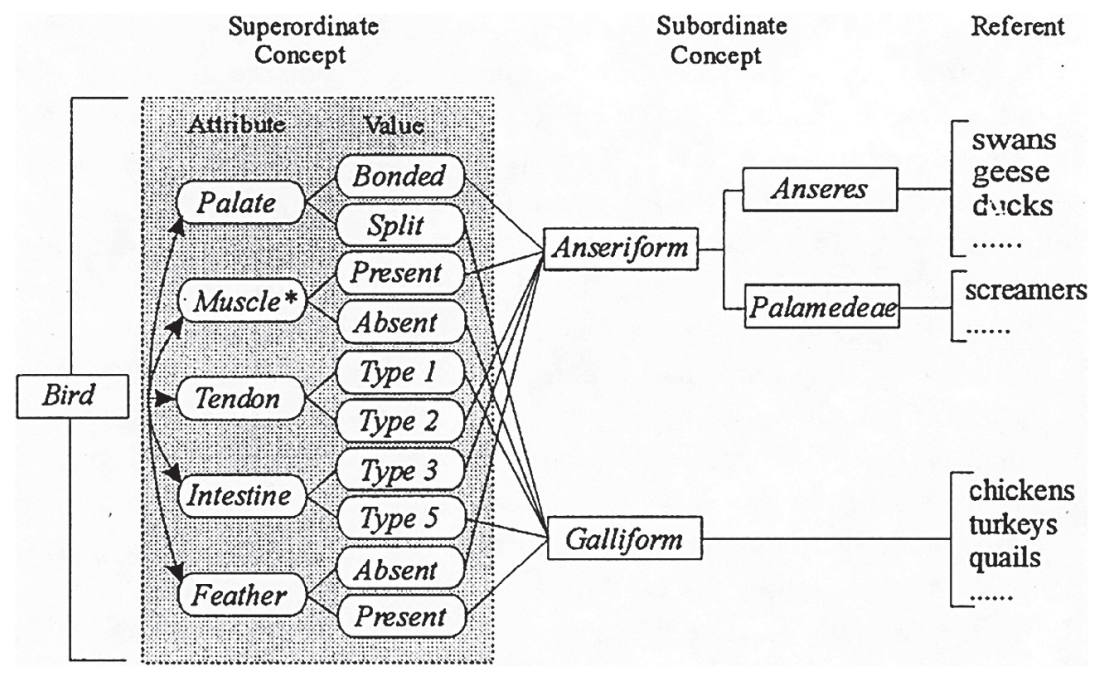

Fig. 3. Partial frame for Gadow’s (1893) bird concept (Chen, 2002)

Except for the attribute feathering arrangement, Gadow's attributes pick out (radically) different morphological features than those in figures 1 and 2. Each attribute, moreover, is connected by a constraint. Constant across the three taxonomies remains the use of body parts, motivated by their cognitive salience (Tversky, Hemenway, 1984; see Chen, 2002: 16f.).

\subsection{Frames and historical scientific change}

The reconstruction and comparison of taxonomies as partial frames has been carried out with particular regard to Kuhn's (1970) incommensurability of taxonomies. ${ }^{1}$ Contra Kuhn, a frame-reconstruction is said to provide some form of comparability. Firstly, applying the frame model facilitates a representation that helps explain why Sundevall's and Gadow's taxonomies are mutually incommensurable: the pair violates Kuhn's no overlap-principle for kind terms (Kuhn, 1993; Chen, 1997). The no-overlap principle can be rendered as "concepts

1 Further applications of the frame model to scientific change can be found, among others, in Andersen, Barker and Chen (1996); Chen et al. (1998); Chen (2003, 2005); Chen and Barker (2000), and the book-length Andersen et al. (2006). Cases range from the wave vs. the particle theory of light, over astronomy and nuclear physics, to the transition from Maxwell's to Einstein's conception of electro-dynamic action. The latter is briefly discussed below. 
belonging to the same subordinate group cannot overlap in their referents" (Barker et al., 2003: 226). This, in turn, shall also help explain why "communication obstacles were bound to occur between the followers of the two systems [... who could not find an equivalent native term with referents that do not overlap those of the foreign one" (Chen, 2002: 9). Secondly, in developing a consensus on the superiority of Gadow's taxonomy over Sundevall's, the scientific community of ornithologist could chose in an instrumentally rational way - or so the reconstructive method supports - because both Gadov and Sundevall used spatial features (body parts). Hence, contrary to the incomparability-interpretation of "incommensurable" - being one the mature Kuhn had come to reject (Chen, 1997; Kuhn, 1983; Hoyningen-Huene, 1993; Zenker, Gärdenfors, 2015) - criteria could have been rationally compared. Therefore, "the preference for spatial features in attribute selection could have functioned as a cognitive platform for the rational comparison of the Sundevall and the Gadow systems $[\ldots]$ " (Chen, 2002: 18).

Allegedly incommensurable taxonomies, thus, may have cut nature along different features, but it were spatial features nevertheless. Such cuts, therefore, need not result in incomparable taxonomies despite violating the no-overlap principle. Consequently, historical transitions between taxonomies that prima facie support the incommensurability thesis (because a given pair of taxonomies is thought to undermine choice-rationality whenever it consists of mutually incomparable alternatives) may - namely upon comparing their frame reconstructions - turn out to be either reconcilable with, or in violation of, one or more standard maxims of choice-rational action, e.g., a mini-max principle (see Zenker, Gärdenfors (2015) on choice-rationality vs. communicative rationality in scientific change). This result, Chen suggests, draws on distinctly cognitive mechanisms.

$[\mathrm{T}]$ axonomic change is rooted deeply in the cognitive mechanisms behind the processes of classification and concept representation. These cognitive mechanisms determine the process of mutual understanding and rational comparison during taxonomic change. In fact, the cognitive platforms for rational comparison identified in our historical cases, that is, compatible contrast sets and attribute lists, were the products of such cognitive mechanisms as the relational assumptions adopted in classification and the preference for body parts developed in concept representation. (Chen, 2002: 19)

We now turn to some aspects of the critical reception of the frame model. 


\subsection{Incomparability and non-translatability}

As Stanford (2008) observes, although they are presumably fine for descriptive purposes, frames do not appear to improve our understanding of incommensurable world-views as cognitive phenomena. But a slightly more drastic consequence might obtain. As far as taxonomic knowledge is concerned, when we employ frames then the incommensurability as incomparability of worldviews is seemingly reconstructed away, while incommensurability as non-translatability of world-views is reconstructively confirmed. After all, as we saw, the frame-reconstruction confirms that historically succeeding taxonomies did overlap in their referents; so its items are not open to a one-to-one translation.

The fact seems to be this: using a frame-model, one reaches a representation where the comparison of the conceptual structures of two or more worldviews consists in nothing but a comparison of their (partial) frames. This allows tracing the requisite constraint violation and observing if, and how, anomalies are resolved in a different frame, or not. If this is a fact, then one may use it in at least two ways. One option is to object, as Stanford did, that the frame model leaves the genesis and the effects of incommensurable world views in the dark; frames merely facilitate a different view on the non-translatability side of the issue - which thus speaks against what Nersessian (1995) dubbed the "cognitive historical approach". But one may also seek to mount the following more far-reaching claim: since the cognitive historical reconstruction renders allegedly incommensurable taxonomies comparable, it follows that incommensurability as incomparability is false as a claim regarding the cognitive representation of concepts. Moreover, if insights into causes and effects of the incommensurability of world-views are needed, then - as far as a cognitive account of conceptual representation is concerned - might not such insights just as well lie outside of it?

One thus suggests that causes and effects of this phenomenon need not be treated as genuine issues of conceptual representation. Instead, incommensurability as non-translatability of world-views, and the communication breakdown that goes along with it, may - perhaps more straightforwardly, too - be explained by citing human imperfections. ${ }^{2}$ Presumably, this option won't sit well with

2 For instance, one might cite psychological deficits, in the sense of having remained, or become, unable to adopt or switch between different views, or strong biases in the sense of no longer considering, e.g., that claims to one ultimate ontology, or a final description of the world, 
everybody. Vis-à-vis the comparability claim, which can be supported both by the frame model and other models of conceptual representation, however, this option may be harder to resist. So, comparability being provided, translatability appears to be a less pressing issue. I take frame models to support that, as a thesis on the incomparability of conceptual structures, incommensurability is a false claim. And as a claim on the non-translatability of world-views, the plausibility of incommensurability can largely, though perhaps not entirely, be accounted for by drawing on factors that do not pertain to human conceptual representation. ${ }^{3}$

\subsection{Conceptual spaces}

The expressive power that frames gain over feature lists is notable, but overall perhaps rather meager. In support of this claim, frames will now be compared to conceptual spaces (Gärdenfors, 2000). The latter is argued to be a more useful model in application to scientific concepts, because measurement-theoretic considerations that, for instance, underlie nominal, ordinal, interval, and ratio scales are native to the model, as it is generally "closer" to mathematics than the other two. Therefore alone, a spatial representation should sit much better with the intuitions of working scientists. Furthermore, reference to the empirical world is of lesser importance; ontological finality is neither implied, nor precluded, by the model. Particularly whether a measurement structure picks out a real structure, or not, isn't a pressing question (see below).

Conceptual spaces provide a geometric or topological model of conceptual knowledge. An assumption that seems basic to the frame model - that concepts are represented primarily symbolically, and only then through the frame-structures themselves - is here discarded. Instead, information is modeled at a level between the symbolic and the subconceptual one. The symbolic forms of mathematical physics, for instance, do therefore not represent concepts, but specify mathematical relational structures. Scientific axioms and laws can therefore be viewed as the symbolic expression of constraints on the distribution of points in a space, a view that will become clearer below.

may be dogmatic, or group-sociological/institutional, in the sense that actors are rationally uncompelled to consider alternatives to some research program that they have been investing in, and have perhaps also profited from.

3 For a more upbeat review of Andersen et al. (2006), see Botteril (2007). For further criticism, see Bird (2012).

\section{O) COGNITIVE SCIENCE}


Past Stevens' (1946) influential work (to whom the above classification of four differentially informative measurement scales goes back), in "mature" measurement theory, mathematical relational structures are embeddable into empirical relational structures, i.e., principally projectable into an ultimate ontology (structures may therefore be called "real"). Stevens did not, in any detail, treat the conditions that such empirical structures should satisfy (see Diez, 1997a: 180). From the point of view of the conceptual spaces model, however, this is fine. After all, the postulated dimensions aren't in any good sense "out there" either. ${ }^{4}$

We now provide a non-technical summary of conceptual spaces. Rigorous treatments include Aisbett and Gibbon (2001) and also Adams and Raubal (2009), an overview of applications of conceptual spaces being provided in Zenker and Gärdenfors (2015).

\subsubsection{Dimensions}

A conceptual space is built up from a number of quality dimensions. Examples include temperature, weight, brightness, pitch, as well as the three ordinary spatial dimensions (height, width, depth). In science, moreover, one regularly finds quality dimensions of an abstract non-sensory character such as mass, force, or energy. The notion of a dimension may be taken literally. Each quality dimension is assumed to be endowed with a certain geometrical structure. Figure 4 illustrates the weight-dimension (one-dimensional with a zero point); it is isomorphic to the half-line of the non-negative numbers. That there are no negative weights is a basic assumption commonly made, e.g., in physics, that is far from trivial; the non-negativity of the weight dimension is a historical contingency. As an ad hoc assumption, for instance, the fire-substance phlogiston (a theoretical entity) had for some time been assumed to have negative weight, and in the late 18th century gave way to the oxygen-account (McCann, 1978; Gärdenfors, Zenker, 2013).

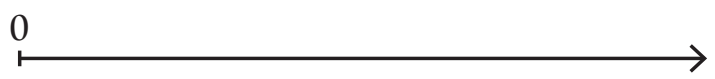

Fig. 4. The weight dimension

4 Some mathematical relational structures have been claimed as being constitutive of empirical relational structures, and so count as methodologically a priori (see, e.g., Friedman 2001). This Neo-Kantian aspect is addressed in Zenker and Gärdenfors (2015). 
As a second example, following Munsell (1915), the cognitive representation of color can be modeled by three dimensions (fig. 5). The first is hue, represented by the familiar color circle (red via yellow to green, blue and back to red). The topological structure of this dimension differs from the dimensions that represents time or weight (which are both isomorphic to the real line). The second dimension is saturation (or chromaticity), ranging from grey (zero color intensity) to increasingly greater intensities; it is isomorphic to an interval of the real line. The third dimension is brightness, varying from white to black; it is a linear dimension with end points. Together, these three dimensions, one with circular and two with linear structure, constitute the color domain which is a subspace of our perceptual conceptual space. This domain is regularly illustrated by the color spindle (two cones attached at their bases). Brightness is shown on the vertical axis; saturation is represented as the distance from the center of the spindle; and hue is represented by the positions along the perimeter of the central circle. The circle at the center of the spindle, moreover, is tilted so that the distance between yellow and white is shorter than that between blue and white.

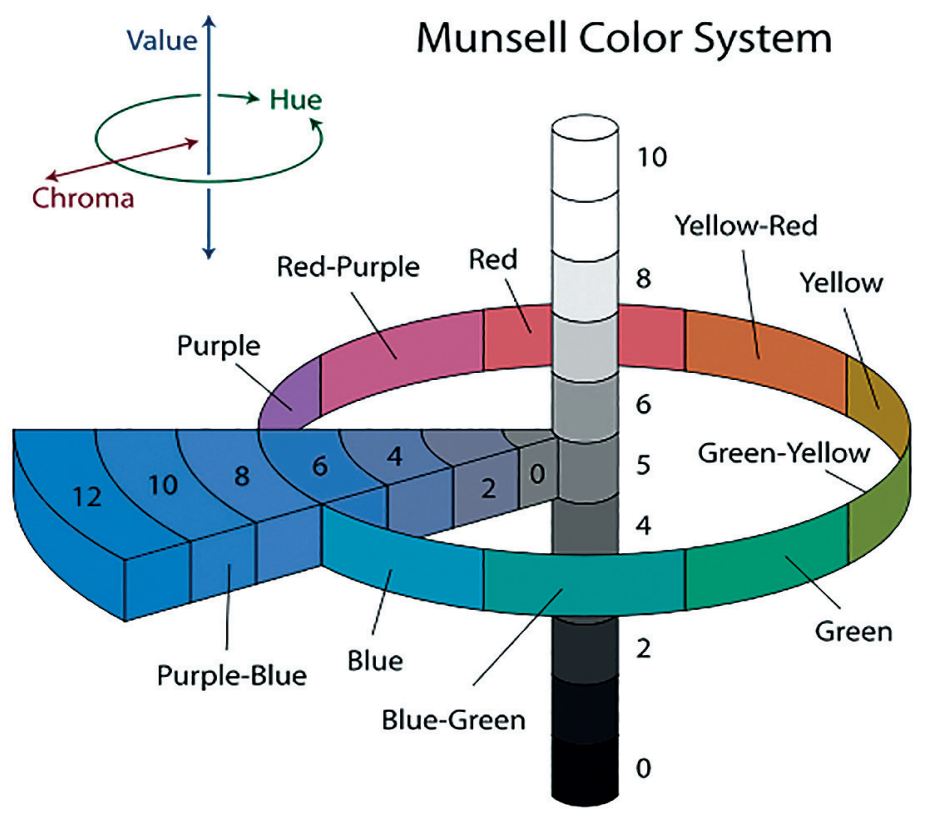

Fig. 5. The Munsell color system

\section{O) COGNITIVE SCIENCE}




\subsubsection{Combinations}

A conceptual space can be defined as a collection of quality dimensions. However, the dimensions of a space should not be viewed as being totally independent. Rather, they are correlated in various ways since the properties of the objects modeled in the space co-vary. For example, in the domain of fruits the ripeness and the color dimensions co-vary. It is presumably not possible to give a complete list of the quality dimensions that make up the conceptual spaces of humans. Some of these dimensions seem to be innate and to some extent "hardwired" (e.g. color, pitch, force, and probably ordinary three dimensional space). Others are presumably learned, yet others are introduced by science (see Gärdenfors, Zenker, 2013). 5

In modeling a scientific concept, the requisite dimensions have to be identified and values that report distances on these dimensions, i.e., a metric (see Berka, 1983: 93), must be assigned. For instance, day and nighttime are standardly modeled by one circular dimension with 24 equally-sized intervals called hours. Before the invention of mechanical clocks, however, the two points on this dimension that separate twelve hours of nighttime from twelve hours of daytime were commonly coordinated locally to sunrise and sunset. As both points shifted, again locally, over the course of one year, their distance changed, thus giving rise to a variable temporal metric which, meanwhile, has given way to one of constant clock intervals. The same occurred in the case of ordinary space, but in the inverse direction - from constant to variable - when the Euclidian metric assumed by Newton was first replace by the Minkowskian metric (used in special relativity) and then the Schwarzschild metric (general relativity), both of which generalize the Euclidian one. It is easy to see that a change of metric leads to a change in the symbolic expressions used to calculate with these distances.

If it is not possible to describe an object fully by assigning a value on one dimension without also assigning a value on another, then such dimensions are said to be integral; otherwise they are called separable. For instance, an object cannot be given a brightness value without also giving it a hue; the pitch of a sound always goes along with its loudness. Or take Newtonian mechanics, where an object is fully described only when it is assigned values on eight dimensions: 3-D space, 1-D time, 3-D force, 1-D mass. ${ }^{6}$

${ }^{5}$ For criticism, see Gauker (2007) and, primarily as to the necessity of positing such spaces, Decock (2006).

${ }^{6}$ Since $F=m a$ holds, some values can be inferred, e.g., for the three force dimensions. 
On these distinctions, the notion of a domain can be defined as a set of integral dimensions separable from all other dimensions. More precisely, domain C is separable from $\mathrm{D}$ in a theory, if and only if the invariance transformations of the dimensions in $\mathrm{C}$ do not involve any dimension from $\mathrm{D}$; and the dimensions of a domain $\mathrm{C}$ are integral, if and only if their invariance class does not involve any other dimension. For instance, until the advent of relativity theories in physics, the three spatial dimensions were separable from the time dimension. So, the spatial coordinates $x, y, z$ were separable from the time coordinate $t$ in Galilean, but not in Lorentz transformations. Moreover, mass is separable from everything else in Newton's theory, but is no longer separable from energy in special relativity (see Gärdenfors, Zenker, 2013).

As the criterion for identifying a domain, we propose the independence of the respective measurement procedures (see Diez, 1997a: 183f.). For instance, in classical mechanics, the measurement of distance and duration (trigonometry and chronometry, respectively) are independent, as light signals are tacitly assumed to propagate instantaneously rather than at finite speed.

\subsection{The conceptual spaces model recovers the frame model}

A comparison between frames and conceptual spaces in case of taxonomic knowledge is straightforward. The main result is that the notions attribute, value, structural invariance and constraint (see above) can be provided with analogues. In particular, the structural invariants and constraints of the frame model arise naturally from the geometry of the conceptual space (e.g., category membership is a matter of occupying regions of a space). It is therefore reasonable to view conceptual spaces as a refinement, or improvement, of the frame model. Using one or the other tool may, at times, be a matter of convenience, related to the complexity of what is to be modeled. For taxonomic knowledge, for instance, conceptual spaces appear over-powerful. Put differently, representing conceptual knowledge at nominal level (through $n$-ary features) is under-complex.

\subsubsection{Analogues}

What on the frame-model is an attribute will correspond to a single dimension or, as the case may be, to combinations thereof. For instance, each color can be represented as a sub-region of the space spanned by hue, saturation and 
brightness, rather than by natural language terms. Further, the value of an attribute corresponds to a point or, as the case may be, to an interval on one or several dimensions. Generally, the metric of a dimension corresponds closest to the attributes' values. On assumption of being an equal distance apart, for instance, the values "large", "medium" and "small" of the attribute "size" will be modeled by an interval-scaled dimension, otherwise by an ordinal scaled one (when this assumption is relaxed), or a nominal-scaled one (when no such ordering relation is assumed), or, in the case of the most informative metric, an ratio scaled one (where the value zero is meaningful, which it is only if what could be measured can in fact be absent). Yet, unlike the conceptual spaces model - where betweenness is meaningful by virtue of the dimensions' geometric properties - nothing in the frame model represents in a motivated way that "medium" is between "big" and "small". Model users know as much, the model doesn't!

The purpose of a constraint, as we had seen, was to rule out, or make unlikely, some among the logically many attribute-value combinations. Constraints thus result from the particular selection of values that define a subordinate category. To mimic this in conceptual spaces, where instances of a concept are represented as points or vectors in an $n$-dimensional space, one may speak of a space's sub-regions being comparatively unpopulated. The notion structural invariant, finally, corresponds to a correlation of dimensions, which means that a number of dimensions represent jointly.

\subsubsection{Similarity as distance}

To appreciate how distances may be exploited in accounting for similarity, reconsider the attributes and values of Sundevall's taxonomy (fig. 2), with abbreviations in brackets:

7 Structural invariants have been interpreted to represent synthetic a priori knowledge, i.e. knowledge about the empirical world that originates in a (taxonomic) structure constitutive of experience, but itself not based in it. Structural invariants, for instance, are claimed to account for such synthetic a priori knowledge claims as: "There are no [normal] birds with legs that attach to their necks" (Barker et al., 2003: 225f.). Denial of this claim may lead a hearer to assume that a speaker does not understand the concept BIRD. The synthetic a priori status of such knowledge can, in principle, be saved in conceptual spaces, assuming one has identified it. At the same time, it remains unclear if singling out these rather than those elements as synthetic a priori is helpful, or necessary (see Zenker, Gärdenfors, 2015). 


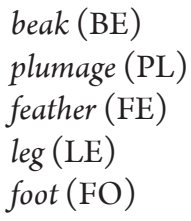

$$
\begin{aligned}
& \text { round (ro), pointed (po) } \\
& \text { course (co), dense (de) } \\
& \text { absent (ab), present (pr) } \\
& \text { skinned (sk), scutate (sc) } \\
& \text { webbed (we), clawed (cl) }
\end{aligned}
$$

One may here treat each attribute as a nominal-scaled dimension. All values are binary, e.g., the beak is either round or pointed; a third value does not apply. So each dimension gives rise to a scale with two parts (nominal order), yielding a total of five integral dimensions. The natural kinds Natarotes, Grallatores, and Gallinea are thus reconstituted as three distinct regions of a mostly unpopulated 5-D space (tab. 1).

Table 1. Comparison of dimensions in Sundevall's taxonomy

\begin{tabular}{|l|l|l|}
\hline \multicolumn{1}{|c|}{ Kind terms } & \multicolumn{1}{|c|}{ Nominal dimensions } & \multicolumn{1}{c|}{ Instances } \\
\hline Natarotes & BE-ro, PL-de, FE-ab, LE-sk, FO-we & swans, geese, ducks \\
Grallatores & BE-po, PL-de, FE-ab, LE-sk, FO-we & herons, screamers, storks \\
Gallinae & BE-po, PL-co, FE-pr, LE-sc, FO-cl & chickens, turkeys, quails \\
\hline
\end{tabular}

Table 1 readily conveys that Natarotes and Grallatores are similar up to the beak-dimension (BE-ro vs. BE-po). Their similarity remains rather hidden in a frame model, but would be immediate in a feature list, or in a conceptual space, here in virtue of Natarotes and Grallatores occupying neighboring regions, or hyperplanes, in a 5-D space. In a frame and a feature list model, moreover, it is unclear how to measure by virtue of the tool employed the comparative distance between Natarotes, Grallatores and Gallinae. In the idiom of conceptual spaces, in contrast, the Gallinae region is maximally distant from the Natarotes region, as it differs on four dimensions from Grallatores. That this distance cannot be expressed more informatively is a result of employing binary features. Generally, moreover, when seeking to express taxonomic difference as distance, then the conceptual space model has been invoked implicitly.

Because the attribute (read: dimension) feather is retained with identical values, one may describe the change from Sundevall's to Gadow's taxonomy as a replacement, or a revision, of four dimensions (cum invariants and constraints). This yields a somewhat trivial, but a no less correct reconstruction of conceptual change. Such is perhaps easier to accept when the incommensurability of

\section{O) COGNITIVE SCIENCE}


world-views is not treated as a genuine issue of conceptual representation (see above). The partial frame of Gadow's new taxonomy, moreover, features five dimensions, not all of which take binary values. One may therefore say that complexity, as measured by the number of dimensions, is not constant. Gadow uses four new dimensions; featuring also one region less, in this respect, his taxonomy is simpler than Sundevall's. The types of intestines (type 3 and 5), by contrast, suggest that complexity has increased. The same seems to hold for the tendon dimension. Prima facie, these dimensions still constitute nominal scales.

By defining change-operations on the dimensions and their mode of combination, the conceptual spaces model may also be applied dynamically. In increasing order of the severity of a revision, these operations are: (i) addition/deletion of laws, ${ }^{8}$ (ii) change in scale/metric, (iii) change in integrality/separation of dimensions, (vi) change in importance (or salience) of dimensions; (iv) addition/ deletion of dimensions (for examples, see Gärdenfors, Zenker, 2010; 2013). A more informative pre- vs. post-change reconstruction would seek to employ the comparative distance between taxonomic items. Relative distance between reconstitutions of dimensional points within (regions of) spaces would thus measure, for instance, whether screamers had become more similar to ducks, or not. Severity of scientific change then comes out as distance between spaces (rather than distance within one and the same space), i.e., as a function of the above types of changes, and some second-order distance measure.

\subsection{Laws as constraints}

In the following, we view scientific laws as symbolic expression of constraints on conceptual spaces. Consequently, historical transitions to a posterior space are in principle continuously reconstructable through a modification of the prior space's dimensions, leaving little room for incommensurability to seriously trouble a cognitive account of scientific conceptual knowledge representation.

The foundations for a theory of measurement in the modern sense arise with Helmholtz (1887), were provided with - some say, insufficient - systematization by Stevens (1946), and have been further developed by Krantz, Luce, Suppes and

8 Note that the first type of change is not strictly a change of the space; a change of law merely effects how the points are distributed in it. 
Tversky $(1971,1989,1990)$. For an overview and the caveat in Steven's work, see $\operatorname{Diez}$ (1997a, b), Hand (2004). When dimensions are fine grained, one approaches scientifically exact measurement. Here, shortcomings in the information conveyed by the frame model's attribute-value structure may be observed that suggest a revision of this model. When attributes are not bi-, but $n$-ary, then any attempt at using frames to model ordering relations between values presumably incurs a revision towards conceptual spaces. For instance, when modelling scientific concepts, e.g., in physics, dimensions tend to (though they need not) be ratio-scales; one will want to make sense of the fact that empirical theories and their mathematical laws commonly depend on, and give rise to, measurement results at this level of scale.

As indicated above, one may also attempt to motivate the symbolic character of scientific laws by virtue of the representational tool. Andersen and Nersessian (2000), for instance, state that they "believe that [frame] analysis can be extended to represent the similarity class of problem situations for nomic concepts" (ibid., 230), i.e., those obeying law-like generalizations. In their electromagnetism example (fig. 6), the Lorentz force-treatment is distinguished from the electromotive force-treatment; frame-style, the attributes conductor, ether, and magnet (values: moving or at rest) are coordinated to the respective force laws, whose symbolic forms however differ strikingly, and implausibly so, as the application situation is identical. (In modern terms, applications pertain to the relative motion of a magnet vis-à-vis that of a conductor.) "[I]n Maxwellian electrodynamics, although the resultant electromagnetic induction is the same whether it is the magnet or the conductor that is moving and the other at rest, these are interpreted as two different kinds of problem situations" (ibid.: 237, italics added). By now, the point of their example will be familiar: by suspending the attribute ether, Einstein's revision of Maxwell's electrodynamics removed a "total overlap" (ibid.) between the two treatments.

As can be observed in fig. 6, the laws are appended, rather than motivated by the frame model. Frames therefore seem to apply to scientific laws without providing insight into their status as symbolic generalizations. Such strikingly different formulae, being standardly treated as evidence of "symbolic rupture" in radical scientific change, however, can also be viewed as the symbolic expressions of constraints over different conceptual spaces. Indeed, scientific laws may be viewed as nothing but the symbolic forms of such constraints. This demotes the importance of laws in scientific change vis-à-vis the dominant view (e.g., Dorato, 2005). 
Consequently, the continuity of mathematical structure that is reconstructively achieved in limiting case reduction (see Batterman, 2003) - an achievement that, following Worrall (1989), structural realists tend to cite as strong evidence in disfavor of incommensurability claims - need not exclusively be treated as a matter of scientific laws or axioms, either. Instead, if empirical theories are primarily characterized through the scale-type of the dimensions - or, more contemporaneously, the admissible transformation of a scale (see Diez, 1997b) - and their mode of combinations (integral vs. separable), then "continuity in scientific change" denotes the continuous generation of a predecessor into a successor space (see Gärdenfors, Zenker, 2013; Zenker, Gärdenfors, 2015).

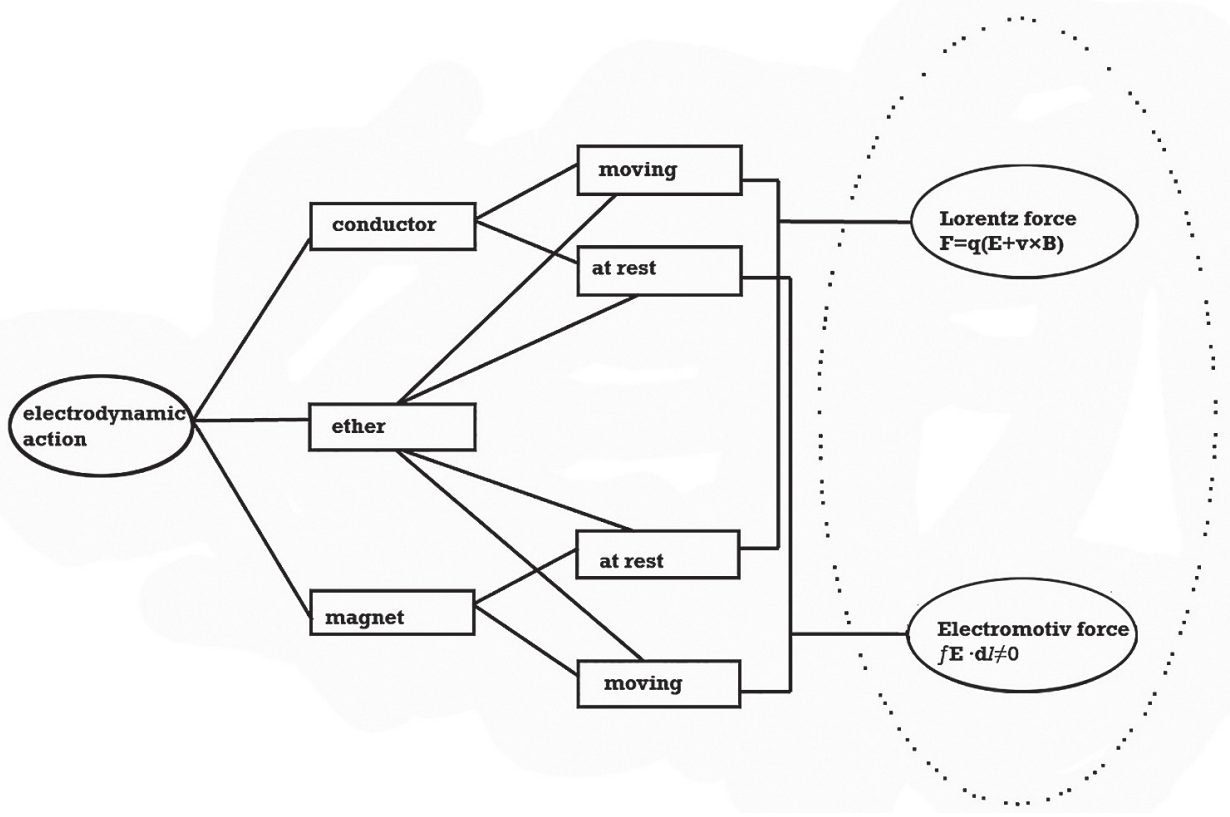

Fig. 6. Partial frame for Maxwellian "electrodynamic action"

(Andersen, Nersessian, 2000: S238)

Zenker and Petersen (2014), for instance, have applied conceptual spaces to the conceptual development of 19th-century fluid dynamics, tracing the changes to the underlying spaces from the Euler equation to the Navier-Stokes equation. On their analysis, the developmental process can be viewed as an instance of normal 
science, in the sense of Kuhn (1970), because the most radical change, the deletion of dimensions, did not occur. Consequently, diachronically prior successful applications of fluid dynamics were always retained as limiting cases of posterior models. (This paper also provides a spatial view on the limiting-case relation.)

To achieve a representation of scientific concepts that is sensitive to the empirical theories in which the concepts feature, but also informed by their historical and potential future dynamics, similarity measures over diachronically related spaces remain wanting. This would hold especially for cases that witness the deletion of dimensions; definitions of such measures remain open to discussion. Extant treatments of conceptual dynamics that project, or transform, conceptual spaces by and large do so through synchronic (see, e.g., Raubal, 2004; Kaipainen, Hautamäki, 2015) rather than diachronic variation. The above types of changes, the definition of a domain, as well as the metric of a dimension should presumably feature as building blocks of such measures.

\section{Conclusion}

The frame model is presumably applicable whenever the conceptual spaces model is. But the latter model gains in applicability to concepts that arise from, and give rise to, exact measurement. Having reviewed the development of feature lists into the frame model, and how to recover frames by conceptual spaces, the latter model's advantages were seen to be gained by making key notions of modern measurement theory native to it. Attempts at achieving as much in the frame model, presumably, would lead to something looking very much like the conceptual space model.

Correspondences between frames and conceptual spaces were pointed out, and it was suggested that using one or the other model may be a mere matter of convenience. For the representation of taxonomic knowledge, in particular, conceptual spaces may well appear over-complex. When addressing the question whether taxonomic items have become more (or less) similar through taxonomical change, one should admit to implicitly presupposing the conceptual spaces model. After all, as we saw, neither frames nor feature lists yield a readily meaningful notion of similarity as geometric distance.

By questioning the assumption that the rationality of a scientific change is inherently a symbolic matter, i.e., has to be demonstrated in symbols, moreover,

\section{O) COGNITIVE SCIENCE}


we have sought to support the claim that conceptual spaces can provide a model for scientific conceptual change across disciplines. Hence, the assumption that a conceptual space is not an intrinsically symbolic model remains indispensable.

\section{Acknowledgements}

This is an extended version of Zenker (2014), originally presented at CTF 2009, University of Düsseldorf, Germany. I would like to thank the audience at this event and the volume's editor, Aleksander Gemel. Research was funded by the Swedish Research Council (VR).

\section{References}

Adams, B., Raubal, M. (2009). A metric conceptual space algebra. In: S. Hornsby, K.C. Claramunt, M. Denis, G. Ligozat (eds). Spatial Information Theory (Proceedings of the 9th International Conference, COSIT 2009, in Aber Wrac'h, France, September 2009), Lecture Notes in Computer Science 5756 (pp. 51-68). Berlin: Springer.

Aisbett, J., Gibbon, G. (2001). A general formulation of conceptual spaces as a meso level representation. Artificial Intelligence 133: 189-232.

Andersen, H., Nersessian N. (2000). Nomic concepts, frames, and conceptual change. Philosophy of Science 67: S224-S241.

Andersen, H., Barker, P., Chen, X. (1996). Kuhn's mature philosophy of science and cognitive psychology. Philosophical Psychology 9: 347-364.

Andersen, H., Barker, P., Chen, X. (2006). The cognitive structure of scientific revolutions. Cambridge: Cambridge University Press.

Barker, P., Chen, X., Andersen, H. (2003). Kuhn on concepts and categorization. In: T. Nickles (ed.). Thomas Kuhn (pp. 212-245). Cambridge: Cambridge University Press.

Barsalou, L. (1992). Frames, concepts, and conceptual fields. In: A.Lehrer, E.F. Kittay (eds). Frames, Fields, and Contrast (pp. 21-74). Hillsdale, NJ: Erlbaum.

Barsalou, L., Hale, C.R. (1993). Components of conceptual representation: From feature list to recursive frames. In: I. Van Mechelen, J. Hampton, R. Michalski, P. Theuns (eds). Categories and Concepts: Theoretical Views and Inductive Data Analysis (pp. 97-144). San Diego, CA: Academic Press.

Batterman, R.W. (2003). The Devil in the Details: Asymptotic Reasoning in Explanation, Reduction, and Emergence. New York, NY: Oxford University Press.

Berka, K. (1983). Measurement: Its Concepts, Theories, Problems. Boston Studies in the Philosophy of Science, vol. 72. Dordrecht: Reidel.

Bird, A. (2012). What can cognitive science tell us about scientific revolutions? Theoria 75: 293-321. 
Botteril, G. (2007). Review of H. Andersen, P. Barker, and X. Chen (2006). The cognitive structure of scientific revolutions. Notre Dame Philosophical Reviews, http://ndpr.nd.edu/ news/25236-the-cognitive-structure-of-scientific-revolutions (accessed: 05.2014).

Chen, X. (1997). Thomas Kuhn's Latest Notion of Incommensurability. Journal for General Philosophy of Science 28: 257-273.

Chen, X. (2002). The 'platforms' for comparing incommensurable taxonomies: A cognitive-historical analysis. Journal for General Philosophy of Science 33 (1): 1-22.

Chen, X. (2003). Object and event concepts: A cognitive mechanism of incommensurability. Philosophy of Science 70: 962-974.

Chen, X. (2005). Transforming temporal knowledge: Conceptual change between event concepts. Perspectives on Science 13: 49-73.

Chen, X., Barker, P. (2000). Continuity through revolutions: A frame-based account of conceptual change during scientific revolutions. Philosophy of Science 67: S208-S223.

Chen, X., Andersen, H., Barker, P. (1998). Kuhn's Theory of Scientific Revolutions and Cognitive Psychology. Philosophical Psychology 11: 5-28.

Decock, L. (2006). A physicalist reinterpretation of 'phenomenal' spaces. Phenomenology and the Cognitive Sciences 5: 197-225.

Diez, J.A. (1997a). A hundred years of numbers. A historical introduction to measurement theory 1887-1990. Studies in History and Philosophy of Science 28: 167-185.

Diez, J.A. (1997b). A hundred years of numbers. A historical introduction to measurement theory 1887-1990. Studies in History and Philosophy of Science 28: 237-265.

Dorato, M. (2005). Why are most laws of nature mathematical? In: J. Faye, P. Needham, U. Scheffler, M. Urchs (eds). Nature's Principles (pp. 55-75). Amsterdam: Springer.

Friedman, M. (2001). Dynamics of Reason. Stanford, CA: CSLI Publications.

Gärdenfors, P. (2000). Conceptual Spaces. The Geometry of Thought. Cambridge, MA: MIT Press.

Gärdenfors, P., Zenker, F. (2010). Using conceptual spaces to model the dynamics of empirical theories. In: E.J. Olsson, S. Enqvist (eds.). Belief Revision meets Philosophy of Science (pp. 137-153). Berlin: Springer.

Gärdenfors, P., Zenker, F. (2013). Theory change as dimensional change: Conceptual spaces applied to the dynamics of empirical theories. Synthese 190: 1039-1058.

Gauker, C. (2007). A critique of the similarity space theory of concepts. Mind and Language 22 (4): 317-345.

Hand, D.J. (2004). Measurement Theory and Practice. London: Arnold.

Helmholtz, H.V. (1887). Zahlen und Messen erkenntnistheoretisch betrachtet. In: H.V. Helmholtz (1920). Schriften zur Erkenntnistheorie (pp. 70-109). Berlin: Springer (transl. in: H.V. Helmholtz (1930). Counting and Measuring (pp. 72-114). Princeton, NJ: Van Noestrand).

Hoyningen-Huene, P. (1993). Reconstructing Scientific Revolutions. Thomas S. Kuhn's Philosophy of Science. Chicago: University of Chicago Press.

Howard, D. (2009). "Let me briefly indicate why I do not find this standpoint natural". Einstein, General Relativity and the Contingent Apriori. In: M. Domski, M. Dickson (eds). 
Discourse on a New Method. Reinvigorating the Marriage of History and Philosophy of Science (pp. 333-355). Chicago and La Salle, ILL: Open Court.

Jaeger, G. (2010). Natural color categorization are convex sets. Lecture Notes in Computer Science 6042: 11-20.

Kaipainen, M., Hautamaki, A. (2015). A perspectivist approach to conceptual spaces. In: F. Zenker, P. Gärdenfors (eds.). Applications of Geometric Knowledge Representation, Synthese Library, vol. X. Dordrecht: Springer.

Krantz, D.H., Luce, R.D., Suppes, P., Tversky, A. (1971, 1989, 1990). Foundations of Measurement, vol. I-III. New York: Academic Press.

Kuhn, T.S. (1970). The Structure of Scientific Revolutions. Chicago, ILL: University of Chicago Press.

Kuhn, T.S. (1983). Commensurability, comparability, and communicability. In: P. Asquith, T. Nickles (eds.). PSA 1982, vol. II (pp. 669-688). East Lansing, MI: Philosophy of Science Association.

Kuhn, T.S. (1993). Afterwords. In: P. Horwich (ed.). World Changes: Thomas Kuhn and the Nature of Science (pp. 311-341). Cambridge, MA: MIT Press.

Kuukkanen, J.-M. (2006). Meaning Change in the Context of Thomas S. Kuhn's Philosophy. Dissertation, University of Edinburgh, http://hdl.handle.net/1842/1259 (accessed: 07.2011).

Labov, W. (1973). The boundaries of words and their meanings. In: C.J. Bailey, W.W. Shuy (eds.). New Ways of Analyzing Variation in English (pp. 340-373). Washington, DC: Georgetown University Press.

McCann, H.G. (1978). Chemistry Transformed: The Paradigm Shift from Phlogiston to Oxygen. Norwood, NJ: Ablex Publishing Corporation.

Minsky, M. (1975). A framework for representing knowledge. In: P. Winston (ed.). The Psychology of Computer Vision (pp. 211-277). New York, NY: McGraw-Hill.

Munsell, A.H. (1915). Atlas of the Munsell Color System. Boston, MA: Wadsworth-Howland.

Nersessian, N.J. (1995). Opening the black box: Cognitive science and history of science. Osiris 10: 195-211.

Raubal, M. (2004). Formalizing conceptual spaces. In: A. Varzi, L. Vieu (eds). Formal Ontology in Information Systems. Proceedings of the Third International Conference (FOIS 2004), vol. 114 (pp. 153-164). Amsterdam: IOS Press.

Rosch, E., Merris, C.B., Gray, W.D., Johnson, D.M., Boyes-Braem, P. (1976). Basic objects in natural categories. Cognitive Psychology 8, 382-439.

Stanford, P.K. (2008). Review of H. Andersen, P. Barker, and X. Chen (2006). The cognitive structure of scientific revolutions. British Journal for the History of Science 41: 116-117.

Stevens, S.S. (1946). On the theory of scales of measurement. Science 103: 677-680.

Thagard, P. (1999). How Scientists Explain Disease. Princeton, NJ: Princeton University Press. Thagard, P. (2009). Review of H. Andersen, P. Barker, and X. Chen (2006). The cognitive structure of scientific revolutions. British Journal for the Philosophy of Science 60: 843-847. 
Taylor, J.R. (2003). Linguistic Categorization. Oxford: Oxford University Press.

Tversky, B., Hemenway K. (1984). Objects, parts, and categories. Journal of Experimental Psychology: General 113: 169-193.

Worrall, J. (1989). Structural realism. Dialectica 43: 99-124.

Zenker, F. (2014). From feature lists via frames to spaces. In: T. Gamerschlag, D. Gerland, R. Osswald, W. Petersen (eds.). Frames and Concept Types: Applications in Language and Philosophy (pp. 69-89). Dordrecht: Springer.

Zenker, F., Gärdenfors, P. (2014). Modeling diachronic changes in structuralism and in conceptual spaces. Erkenntnis 79 (8): 1547-1561.

Zenker, F, Gärdenfors, P. (2015). Communication, rationality, and conceptual changes in scientific theories. In: F. Zenker, P. Gärdenfors, (eds.). Applications of Geometric Knowledge Representation, Synthese Library, vol. X. Dordrecht: Springer.

Zenker, F., Petersen, G. (2014). From Euler to Navier-Stokes: A spatial analysis of conceptual changes in 19th-century fluid dynamics. International Studies in the Philosophy of Science 28 (3): 1-19. 